SPECIAL ISSUE EDITORIAL

\title{
Understanding Babinski’s anosognosia: 100 years later
}

\author{
Paul M. Jenkinson \\ Department of Psychology \\ University of Hertfordshire \\ \& \\ Aikaterini (Katerina) Fotopoulou \\ Research Department of Clinical, Educational and Health Psychology \\ University College London
}

Address for correspondence:

Paul Jenkinson: p.jenkinson@herts.ac.uk

Aikaterini Fotopoulou: a.fotopoulou@ucl.ac.uk 


\section{Introduction of a new term}

In 1914 Joseph Babinski performed a common, scientific act that nevertheless had far-reaching consequences: he presented a paper in which he introduced a new

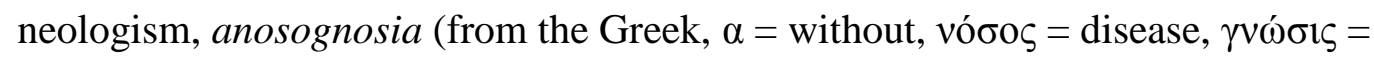
knowledge) to describe behaviours previously noted by himself and others (see Berrios \& Markova, this issue). In the 1914 paper, Babinski observed how some patients with left hemiplegia were "unaware of or seem to be unaware of the existence of the paralysis which affects them” (Babinski, 1914, see translation by Langer \& Levine, this issue, pp. XXX). The paper was accompanied by many fascinating commentaries (see translation by Langer \& Levine, this issue) and anosognosia has since been at the focus of much medical and scientific research, including two dedicated volumes of collected essays (Prigatano, 2010; Prigatano \& Schacter, 1991) and hundreds of scientific papers (see Nurmi \& Jehkonen, this issue, for a review). In the years since Babinski, definitions have been modified and extended to include unawareness of numerous disorders, multiple domains, and now includes several subtypes. This year marks the $100^{\text {th }}$ anniversary of Babinski introducing the term, and this special issue aims to provide a $21^{\text {st }}$ century equivalent of Babinski’s original work: a reference point for recent developments in the field, and a fertile environment for the generation of novel hypotheses.

2.1. The changing face of anosognosia research: Current Answers to 100-year-old Questions

Since 1914 the study of anosognosia has undergone considerable development. First, the term anosognosia has been broadened in scope to encompass an array of failures to appreciate the presence or severity of various neurological deficits, such as 
hemianaesthesia or apraxia, following focal lesions, as well as diffuse brain damage (see in this issue Canzano, Scandola, Perigo, Aglioti \& Moro; Pia et al.; and Ronchi \& Vallar). In recent years, the condition has also been studied in the context of degenerative diseases like Alzheimer's disease (see Starkstein; and Spalletta et al., this issue) and Parkinson’s disease (Amanzio et al., 2010; Jenkinson, Edelstyn, Stephens, \& Ellis, 2009).

Second, the methods used to study the neural basis of anosognosia have changed dramatically. Babinski could only speculate about the lesion location in his patients based on their left hemiplegia. Improvements in structural neuroimaging methods have since allowed not only lesion identification in individual patients, but also group lesion mapping studies to identify the specific, right hemisphere lesions selectively associated with AHP and as opposed to other aspects of the righthemisphere syndrome (Berti et al., 2005; Karnath, Baier, \& Nägele, 2005). In turn, the limitations of such methods and the association between anosognosia and the left hemisphere (Cocchini, Beschin, Cameron, Fotopoulou, \& Della Sala, 2009) are currently been tackled by functional neuroimaging studies, as the current issue exemplifies (see Gandola et al.; Baier et al.). These changes in neuroscientific methodology are coupled with improvements in behavioural methods, which have enriched clinical observations and case studies with well-controlled, psychophysical experiments in larger groups of patients (see for example, Saj, Vocat \& Vuilleumier, and Besharati et al., this issue, as well as Fotopoulou, 2014; Jenkinson \& Fotopoulou, 2010; for reviews).

Last but certainly not least, these developments have been accompanied by progress in the theoretical conceptualisation of anosognosia. Remarkably, it took 100 years to answer a lot of the questions posed by Babinski and his contemporaries (see 
Langer \& Levine for the rich set of commentaries accompanying Babinki’s paper) and in fact several, critical components of the syndrome seem to be elucidated only recently, while others are debated to this day. For example, Babinski's two cases differ in that the first patient remains silent when asked to move the affected arm and tries to avoid such questions, while the second claims to have moved in front of the examiner. This difference between general false beliefs (delusions) about one’s motor abilities and more specific claims about moving after 'confrontation' techniques has long been included in clinical, diagnostic assessments of anosognosia (e.g. Bisiach et al., 1986), but it is only in the last 15 years that the presence of such 'illusory movements' has been experimentally verified (Feinberg, Roane, \& Ali, 2000; Fotopoulou et al., 2008), influencing theories about non-veridical motor awareness (Berti et al., 2007).

Another example is the role of motivation in anosognosia. Babinski and his contemporaries portray a profound dualism in their thinking. For example, Babinski notes that the patients' relatives considered the unawareness as useful and had asked the doctors not to challenge the patients' tranquillity. He thus wonders whether anosognosia is motivated by vanity or self-esteem, or whether it is 'real'. In his commentary, M. Henry Meige writes "while it is common to see an aphasic get angry, to bemoan an unsuccessful attempt at speech, the hemiplegic, in contrast, complains more rarely of the inertia of his arm or leg. Is it resignation, a wish to hide from himself or others a defect that afflicts him? It is possible, in certain cases; but in others one is faced with a true psychopathological problem” (see Langer \& Levine, this issue, pp. XXX). This absolute contrast between a psychological wish to be healthy and to deceive the self and others accordingly, and a neurological condition that deprives the person of knowledge into their abilities, is a contrast that colours 
most explanations of anosognosia during the 20th century. It is only in the past 15 years that integrative perspectives have emerged, linking emotion and motivation more directly to the brain damage seen in anosognosic patients. In the current issue, papers by Turnbull, Fotopoulou and Solms, and Besharati et al. exemplify such integrative approaches, challenging rigid distinctions between the psychological and neural levels of explanation.

As we outline in the next section, similar progress is currently noted in relation to several facets of the syndrome. In this issue, contributions from leading researcher in the field have been gathered together in a unique collection of reviews and empirical papers on anosognosia, aiming to give a comprehensive view of the current state of knowledge in the field. Remarkably, despite the impressive research developments exemplified by these papers, Babinski and his colleagues had raised a number of questions that remain unanswered. We thus briefly review the aforementioned progress in section 2.2 below, before going on to summarise the questions that remain unanswered, as well as the novel ones that are beginning to appear after 100 years of research into the syndrome.

\subsection{In this issue}

In the first part of this issue, Langer and Levine provide the first unabridged English translation of Babinski’s original paper and its accompanying commentaries allowing non-French speakers the opportunity to read in full the original ideas of Babinski and his colleagues. In a specialist historical review, Berrios and Markova then consider the early, mostly European and at times, previously untranslated case studies on anosognosia and put forward their views on the historical 'construction' of anosognosia as a unique clinical disorder. In the spirit of Babinski’s original paper, 
Prigatano goes on to describe the pattern of impaired self-awareness observed in clinical practice, giving rich clinical descriptions of four patients with various disturbance of awareness. In a similar vein, Turnbull et al. use a clinical vignette to revisit the history of the rise and fall of one of the older hypothesis of anosognosia, namely the motivational hypothesis, and to provide a modern interpretation of the syndrome that combines neurocognitive deficits and emotional factors in a single formulation. Heilman's thorough review also highlights the requirement to consider a combination of different causes, but his extensive coverage of studies using the WADA test and other experimental procedures calls for greater specificity and portrays a different view of motivational factors. These careful analyses of the mechanisms that may cause anosognosia are followed by a systematic review of the incidence and assessment of anosognosia by Nurmi and Jehkonen. They provide a comprehensive picture of how research has evolved during the past 35 years, highlighting how the focus of anosognosia research has extended over this period to include a wider variety of deficits, with anosognosia now firmly established as a multifaceted syndrome.

Five subsequent papers exemplify the variety of new experimental and neuroscientific methodologies brought to the study of anosognosia in later years, ranging from functional neuroimaging to carefully conducted, experimental paradigms of relevant behaviours in neurological patients and healthy controls. The role of faulty action monitoring as a cause of anosognosia has become increasingly recognised, and papers by Saj et al., and Gandola et al. examine new facets of this explanation, using carefully controlled experimental paradigms and neuroscientific methods such as transcranial direct current stimulation (tDCS). They are followed by a report by Baier et al., of the first ever functional imaging (fMRI) study of 
anosognosia following unilateral left-hemisphere stroke, in which language lateralisation was reversed to the right-hemisphere. A study of anosognosia for hemiplegia by Besharati et al. looks beyond the motor accounts, and at the potential role of emotional and motivational factors in anosognosia, providing empirical confirmation for some of the claims made by Turnbull et al. Finally, McKay, Buchmann, Germann, Yu and Brugger introduce the novel idea of studying 'nosognosia' in healthy people, in order to identify the normal function that is disrupted in patients with anosognosia.

A further three studies examine anosognosia for different domains following stroke, thereby highlighting a major development in anosognosia research: i.e. the existence of multiple, modular awareness systems that can give rise to different, specific forms of anosognosia. First, Canzano et al. suggest a new form of anosognosia in which patients are unaware of apraxia, while, Pia et al. continue the theme with an examination of anosognosia for hemianaesthesia (i.e. loss of tactile sensibility on the contralesional body side). Ronchi and Vallar then examine unawareness of unilateral spatial neglect, demonstrating that awareness even within a given domain (i.e. visual-spatial ability) can vary considerably.

In the final papers of the special issue we examine anosognosia in relation to Alzheimer's disease, and consider a specific disorder of body ownership unawareness, whose relation with anosognosia remains debated. First, Starkstein provides a valuable summary of the advances made in research into anosognosia in Alzheimer's disease, covering key issues relating to diagnosis, frequency, proposed mechanisms and clinical correlates. The complexity of the issue as highlighted by this review, and the complex research task that lie ahead, are suitably illustrated by the next paper by Spalletta et al., who examine the neuroanatomical correlates of impaired awareness in 
patients with amnestic mild cognitive impairment. Finally, Feinberg and Venneri focus on aberrant body ownership following right-hemisphere stroke in their review of somatoparaphrenia. Their paper examines the neuroanatomical and functional mechanisms responsible for this rare disorder, and provides unique neuropsychological insights into the fundamental constituents of the bodily self.

\section{Beyond Babinski and this Issue: New Questions for the Next 100 Years}

Overall, the papers in this special issue highlight the diverse and significant advances that have been made in anosognosia research over the past 100 years. As aforementioned, several of the questions raised during Babinski’s time have been addressed, while others remain debated today. However, most interestingly, this issue also offers a view of new questions and novel directions for research. We summarise below what we feel are the three major directions that $21^{\text {st }}$ century research on anosognosia is likely to take.

One of the greatest outstanding challenges is the need to develop an adequate, multifaceted explanation of anosognosia across patients and disorders. A century of research has found anosognosia to be ever more complex - and attempts to develop a 'single deficit' explanation have been accordingly fruitless and ultimately futile. Future research must provide a unified framework of normal brain function that is able to explain the dynamic, heterogeneous and multifaceted nature of anosognosia. Recent cognitive accounts of anosognosia have responded by proposing models that combine multiple top-down and bottom-up factors (e.g. Davies, Davies, \& Coltheart, 2005; Mograbi \& Morris, 2013; Vuilleumier, 2004), whereas a 'predictive coding' account (see Fotopoulou, 2012; \& in press) draws on a 'free energy' computational, model of normal brain function (Friston, 2009) to unify such factors under one 
common operating principle. Although further empirical research is needed to test these new models, they hold the potential of asking new, better questions about the nature of anosognosia. For example, future studies could clarify the neural and psychological reasons why in certain patients anosognosia is specific to particular domains, while in others it can affect several deficits; or why in certain patients anosognosia disappears after a few days, while in others it last for years.

Developing an adequate explanation of anosognosia in the future will also depend on how we define and examine fundamental properties of the mind, such as self-consciousness. Recent cognitive neuroscience research has distinguished different components of bodily self-consciousness (i.e. agency and body ownership), and examined these using transient multisensory illusions and functional neuroimaging in healthy controls (e.g. Tsakiris, Longo, \& Haggard, 2010). This work provides a new and productive way of studying self-consciousness; however, the phenomenally elusive constituents of the self remain difficult to examine using such illusions. By contrast, patients with anosognosia and related disorders provide a more stable and rich source of data regarding the neurocognitive bases of selfconsciousness. Using advanced structural and functional brain imaging to study anosognosia can, therefore, reveal both the mechanisms underlying the disorder and neuroanatomical correlates of self-consciousness. It follows, that it may soon be possible to relate anosognosia to other awareness concepts such as the concept of 'insight' as used in psychiatry, as well as the lack of illness appreciation as observed in eating and functional disorders.

Studying the neuroanatomical bases of anosognosia may also provide new approaches for rehabilitation and restoration of awareness. This raises several important questions for the future: can we predict who will develop anosognosia and 
how the condition will evolve over time? Can we use this information to personalise treatment and ensure the best prognosis? And perhaps most importantly - can we restore normal awareness? Existing research has already proven successful at identifying the clinical and anatomical predictors of recovery from other strokeinduced deficits, such as aphasia (Forkel et al., 2014) and visuospatial neglect (Karnath, Rennig, Johannsen, \& Rorden, 2011). Similarly, several studies in this issue have identified the neuroanatomical correlates of anosognosia (e.g. Baier et al.; Besharati et al.; Pia et al.; and Spalletta et al.), and show that a change in awareness can be produced using simple behavioural or neuromodulatory techniques (e.g. Besharati et al.; Gandola et al.; and Ronchi \& Vallar). These findings are certainly promising; however, further experimental studies, and randomised controlled trials are needed to identify the optimal methods for predicting recovery or restoring awareness.

Importantly, future research that seeks to understand and develop treatments for anosognosia may involve a paradigm shift that also holds unprecedented potential for: (i) understanding the ways in which the brain changes plastically or reorganises its higher order cognitive functions following brain damage; and (ii) exemplifying the interrelation between the mind, body, brain and person. The hope is the research into anosognosia can pave the way for a more integrative, multidisciplinary approach to brain damage, which considers its effects on the brain, the individual, and the environment. Thus, the next 100 years of anosognosia research may serve as an indispensable window of insight into the neurobiology of a fundamental property of the mind, namely self-consciousness. 


\section{REFERENCES}

Amanzio, M., Monteverdi, S., Giordano, A., Soliveri, P., Filippi, P., \& Geminiani, G. (2010). Impaired awareness of movement disorders in Parkinson's disease. Brain and Cognition, 72(3), 337-46. doi:10.1016/j.bandc.2009.10.011

Babinski, J. (1914). Contribution à l'étude des troubles mentaux dans l’hémiplégie organique cérébrale (anosognosie). Revue Neurologique (Paris), 27, 845-848.

Berti, A., Bottini, G., Gandola, M., Pia, L., Smania, N., Stracciari, A., ... Paulesu, E. (2005). Shared cortical anatomy for motor awareness and motor control. Science, 309(5733), 488-491. doi:10.1126/science.1110625

Berti, A., Spinazzola, L., Pia, L., Rabuffetti, M., Haggard, P., Rossetti, Y., \& Kawato, M. (2007). Motor awareness and motor intention in anosognosia for hemiplegia. In Sensorimotor foundations of higher cognition (pp. 163-181). Oxford: Oxford University Press.

Bisiach, E., Vallar, G., Perani, D., Papagno, C., Berti, A., \& Neurologica, C. (1986). Unawareness of disease following lesions of the right hemisphere: Anosognosia for hemiplegia and anosognosia for hemianopia. Neuropsychologia, 24(4), 471482.

Cocchini, G., Beschin, N., Cameron, A., Fotopoulou, A., \& Della Sala, S. (2009). Anosognosia for motor impairment following left brain damage. Neuropsychology, 23(2), 223-30. 
Davies, M., Davies, A. A., \& Coltheart, M. (2005). Anosognosia and the Two-Factor Theory of delusions. Mind and Language, 20(2), 209-236.

Feinberg, T. E., Roane, D. M., \& Ali, J. (2000). Illusory limb movements in anosognosia for hemiplegia. Journal of Neurology, Neurosurgery, and Psychiatry, 68(4), 511-3.

Forkel, S. J., Thiebaut de Schotten, M., Dell’Acqua, F., Kalra, L., Murphy, D. G. M., Williams, S. C. R., \& Catani, M. (2014). Anatomical predictors of aphasia recovery: A tractography study of bilateral perisylvian language networks. Brain, 137(7), 2027-2039.

Fotopoulou, A. (in press). The Virtual Bodily Self: The Mentalisation of the Body as Revealed in Anosognosia for Hemiplegia. Consciousness and Cognition.

Fotopoulou, A. (2012). Illusions and delusions in anosognosia for hemiplegia: From motor predictions to prior beliefs. Brain : A Journal of Neurology, 135(5), 13446. doi:10.1093/brain/aws094

Fotopoulou, A. (2014). Time to get rid of the "Modular" in neuropsychology: A unified theory of anosognosia as aberrant predictive coding. Journal of Neuropsychology, 8(1), 1-19. doi:10.1111/jnp.12010

Fotopoulou, A., Tsakiris, M., Haggard, P., Vagopoulou, A., Rudd, A., \& Kopelman, M. (2008). The role of motor intention in motor awareness: An experimental study on anosognosia for hemiplegia. Brain, 131(12), 3432-42.

Friston, K. (2009). The free-energy principle: a rough guide to the brain? Trends in Cognitive Sciences, 13(7), 293-301. doi:10.1016/j.tics.2009.04.005 
Jenkinson, P. M., Edelstyn, N. M. J., Stephens, R., \& Ellis, S. J. (2009). Why are some Parkinson disease patients unaware of their dyskinesias? Cognitive and Behavioral Neurology, 22(2), 117 121. doi:10.1097/WNN.0b013e3181a722b0

Jenkinson, P. M., \& Fotopoulou, A. (2010). Motor awareness in anosognosia for hemiplegia: Experiments at last! Experimental Brain Research, 204(3), 295-304. doi:10.1007/s00221-009-1929-8

Karnath, H.-O., Baier, B., \& Nägele, T. (2005). Awareness of the functioning of one’s own limbs mediated by the insular cortex? The Journal of Neuroscience, 25, 7134-7138.

Karnath, H.-O., Rennig, J., Johannsen, L., \& Rorden, C. (2011). The anatomy underlying acute versus chronic spatial neglect: A longitudinal study. Brain, 134(3), 903-912.

Mograbi, D. C., \& Morris, R. G. (2013). Implicit awareness in anosognosia: clinical observations, experimental evidence, and theoretical implications. Cognitive Neuroscience, 4(3-4), 181-97. doi:10.1080/17588928.2013.833899

Prigatano, G. P. (Ed.). (2010). The study of anosognosia. New York: Oxford University Press.

Prigatano, G.P. \& Schacter, D.L. (Eds.). (1991). Awareness of deficit after brain injury: Clinical and theoretical issues. New York: Oxford University Press. 
Tsakiris, M., Longo, M. R., \& Haggard, P. (2010). Having a body versus moving your body: Neural signatures of agency and body-ownership. Neuropsychologia, 48(9), 2740-2749. doi:DOI 10.1016/j.neuropsychologia.2010.05.021

Vuilleumier, P. (2004). Anosognosia: The neurology of beliefs and uncertainties. Cortex, 40, 9-17. 